\title{
Board Games for Quantum Computers
}

\author{
Biao $\mathrm{Wu}$ (吴骵) ${ }^{1,2,3}$ Hanbo Chen(陈汉博), ${ }^{1}$ and Zhikang Luo(罗智康 $)^{4}$ \\ ${ }^{1}$ International Center for Quantum Materials, \\ School of Physics, Peking University, 100871, Beijing, China \\ ${ }^{2}$ Wilczek Quantum Center, School of Physics and Astronomy, \\ Shanghai Jiao Tong University, Shanghai 200240, China \\ ${ }^{3}$ Collaborative Innovation Center of Quantum Matter, Beijing 100871, China \\ ${ }^{4}$ College of Chemistry and Molecular Engineering, \\ Peking University, 100871, Beijing, China
}

(Dated: April 20, 2020)

\begin{abstract}
Scalable board games, including Five in a Row (or gomoku) and weiqi (or go), are generalized so that they can be played on or by quantum computers. We adopt three principles for the generalization: the first two are to ensure that the games are compatible with quantum computer and the third is to ensure that the standard classical games are the special cases. We demonstrate how to construct basic quantum moves and use them to set up quantum games. There are three different schemes to play the quantized games: one quantum computer with another quantum computer (QwQ), two classical computer playing with each other on one quantum computer (CQC), and one classical computer with another classical computer $(\mathrm{CwC})$. We illustrate these results with the games of Five in a Row and weiqi.
\end{abstract}




\section{INTRODUCTION}

There are many interesting board games. Chess, weiqi (or go as usually called in English), and Five in a Row (or gomoku) are among the most popular. In particular, both weiqi and FIR (Five in a Row) are played on scalable square boards (see Fig. 1.1), where two players take turn to place black and white stones on the board. Following the convention of quantum information, we call the two players Alice and Bob who play, respectively, white stones and black stones. In this work, we focus our attention on these scalable board games and discuss how to generalize them so that they can be played by or on quantum computers with intrinsic quantum moves.

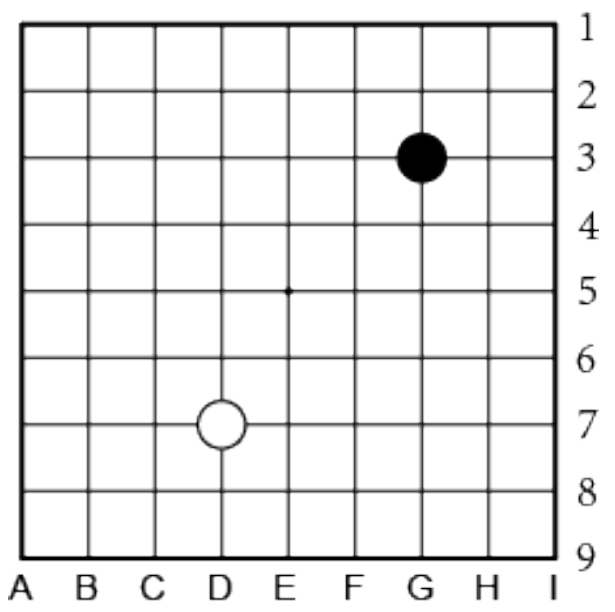

FIG. 1.1: Scalable board. The board is labelled horizontally by Roman letters and vertically by Arabic numbers so that each point (or intersection) is specified by coordinates. For example, the coordinates of the central point are $(E, 5)$. The game is played by two players who take turn to place black and white stones on the points of the board. This board has a grid of $9 \times 9$. Even though the usual boards used in Five in a Row (FIR) and weiqi have grids of $15 \times 15$ and $19 \times 19$, respectively, both games can be played on boards of other sizes.

The most popular model of quantum computer consists of a set of quantum logical gates operating on an array of quantum bits (or qubits) [1]. As there are three states, black stone, white stone, and unoccupied, at points (intersections of grid lines), the board, such as the one in Fig. 1.1, can be considered as an array of quantum trits (or qutrits). It is therefore natural and convenient for us to use a different model of quantum computer, where a set of quantum logical gates operate on an array of qutrits [2]. 
We will begin by considering the principles for designing scalable board games for quantum computers. First of all, the moves should be unitary and made of a number of quantum gates. Moreover, as the board is scalable, the number of quantum gates in a move should not be exponentially large. We review the basic moves in the classical games of FIR and weiqi and show that they can be represented by unitary transformations, i.e., quantum gates. We then construct various quantum moves and use them to design games for quantum computer. Due to its simplicity, we use FIR as an example to discuss three different schemes to play quantum game: one quantum computer with another quantum computer $(\mathrm{QwQ})$; two classical computers playing with each other on a quantum computer (CQC), and one classical computer with another classical computer $(\mathrm{CwC})$. For the third scheme, as a classical computer can not store faithfully a quantum state in a Hilbert space of exponentially large dimension, one may have to impose an upper limit on the number of superposed games. The game of weiqi is much more complicated. We find that its quantum version may never finish when played by or on quantum computer.

\section{PRINCIPLES OF QUANTUM GAMES}

In the circuit model of quantum computer, there are a set of universal quantum gates that operate on an array of qubits or qutrits [1, 2]. These quantum gates have two important features: (1) they are unitary transformations and (2) they involve only one or two qubits (or qutrits). In addition, when Hadamard gate and phase-related gates are not used, a quantum computer becomes a reversible classical computer. To respect these three features, we should adhere to the following three principles for designing scalable board games played on or by quantum computers.

P1. All the moves are unitary transformations;

P2. All the moves involve only finite number of correlated quantum bits;

P3. When it is limited to a true subset of all the moves, the game becomes classical.

For a game to be played on or by quantum computers, moves must be quantum gates or combinations of various quantum gates. Since all the quantum gates are unitary transformations, all the moves must be unitary. To explain the principle $\mathbf{P 2}$, let us suppose there is 
a move that involves $m$ correlated qubits and the number $m$ is proportional to $n$, the total number of points on the board. This move corresponds to a generic $3^{m} \times 3^{m}$ unitary matrix, which is usually a product of about $3^{m}$ to $3^{2 m}$ basic quantum gates upon decomposition [1]. This implies that it would take an exponentially long time to execute a single move of this kind. This is not reasonable and so we have the principle P2. The word "correlated" is crucial here. For a move that involves $m$ uncorrelated quantum bits, it can be expressed as a combination of $m$ one-qubit quantum gates (see, for example, the capture move in Section III). The principle $\mathbf{P} 2$ is also related to the $k$-local property of Hamiltonians that are used to model quantum computers [3]. The principle P3 is obvious. In this work, we only consider quantum board games that are generalized from the well-known classical games. In such cases, the principle P3 means that we recover the standard classical games when it is limited to a true subset of all the moves. It would be interesting if one can design a new quantum game that is not connected to any known classical game. In this case, when the principle P3 is applied to this new quantum game, we would have a new classical game.

Just like in any classical game, the rules of a quantum game should be fair to all players, for example, the player making the first move should have as little advantage as possible. Since this is not unique to quantum games, this is not listed in the above principles.

\section{REVIEW OF CLASSICAL GAMES}

We now review the moves in the classical games of FIR and weiqi. We show that all of them are special cases of quantum moves and obey the principles $\mathbf{P} \mathbf{1}$ and $\mathbf{P} 2$. We use Dirac notations to denote the state at each point and the whole board configuration. For example, $|\mathbf{O}\rangle,|\bigcirc\rangle$, and $|U\rangle$ denote, respectively, a black stone, a white stone, and unoccupied at a given point. For the whole board, one should specify the states for all the points on the board. However, for simplicity, we will not specify the unoccupied points explicitly in a ket (or bra) state. For instance, the board configuration in Fig.1.1 can be denoted as $\left|\bigcirc_{G 3} \bigcirc_{D 7}\right\rangle$, indicating that there are a black stone at the point $(G, 3)$ and a white stone at the point $(D, 7)$ while no stones elsewhere. From now on, only in some special cases we will specify unoccupied points explicitly in ket (or bra) states.

In the standard games of FIR and weiqi, there is one basic move, placing a black or white stone on an unoccupied point. Placing a black stone and a white stone on an unoccupied 
point can be mathematically expressed as

$$
|\bigcirc\rangle=X^{b}|U\rangle, \quad|\bigcirc\rangle=X^{w}|U\rangle
$$

where $X^{b}$ and $X^{w}$ are transformations that can be put into matrix forms. If we let

$$
|\boldsymbol{\gamma}\rangle=\left(\begin{array}{l}
1 \\
0 \\
0
\end{array}\right), \quad|U\rangle=\left(\begin{array}{l}
0 \\
1 \\
0
\end{array}\right), \quad|\bigcirc\rangle=\left(\begin{array}{l}
0 \\
0 \\
1
\end{array}\right)
$$

we have

$$
X^{b}=\left(\begin{array}{lll}
0 & 1 & 0 \\
1 & 0 & 0 \\
0 & 0 & 1
\end{array}\right), \quad X^{w}=\left(\begin{array}{lll}
1 & 0 & 0 \\
0 & 0 & 1 \\
0 & 1 & 0
\end{array}\right)
$$

It is interesting and important to note that $X^{b}$ and $X^{w}$ also remove stones from the board, i.e.,

$$
|U\rangle=X^{b}|\mathbf{O}\rangle, \quad|U\rangle=X^{w}|\bigcirc\rangle
$$

Both $X^{b}$ and $X^{w}$ are clearly unitary transformations and belong to a family of one-qutrit quantum gates [2].

There is a special move in the game of weiqi, capture of stone(s). Two examples are given in Fig 3.1. In the first example, after Bob places a black stone at the point $(E, 6)$, the white stone at the point $(E, 5)$ is captured and removed from the board. We can mathematically express the capture as

$$
\left|\bigcirc_{E 5} \bigcirc_{F 4} \bigcirc_{F 6} \bigcirc_{G 5}\right\rangle=X_{F 5}^{w} X_{F 4}^{b}\left|\bigcirc_{E 5} \bigcirc_{F 6} \bigcirc_{G 5} \bigcirc_{F 5}\right\rangle
$$

Although Bob placed only one stone on the board, due to the mandatory removal of the captured white stone, this move consists of two one-qutrit quantum gates, $X_{F 4}^{b}$ and $X_{F 5}^{w}$. In many situations, multiple stones can be captured simultaneously. For the second example in Fig, 3.1, after Bob places one black stone at the point $(E, 6)$, three white stones are captured and removed. Mathematically, it can be expressed as

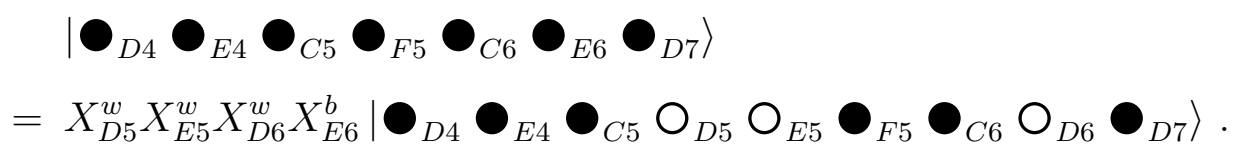

In this case, one capture moves consist of four one-qutrit quantum gates. In the most extreme case where one player's stones are all captured at the late stage of the game, we 
(a1)

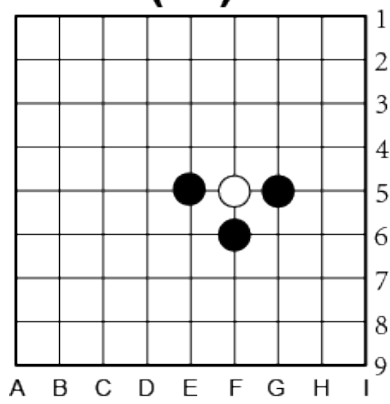

(b1)

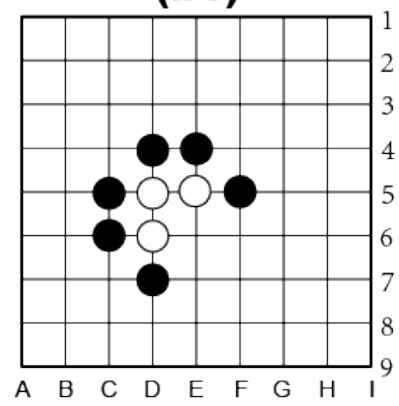

(a2)

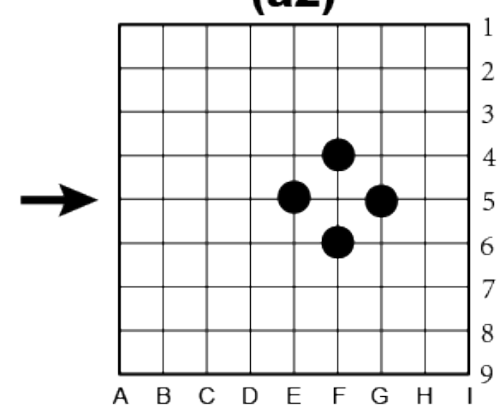

(b2)

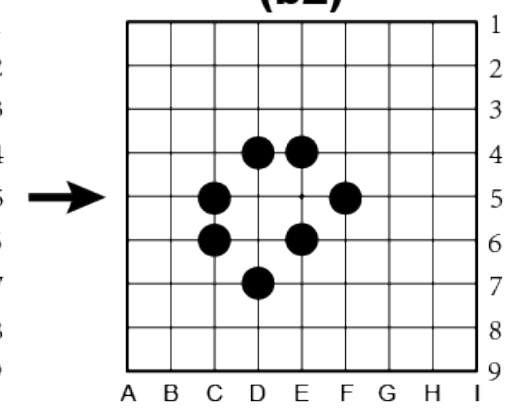

FIG. 3.1: Two examples of capture in the game of weiqi. After one more black stone is placed on the board, the white stones is(are) captured and removed from the board.

would have a capture move consisting of a number of one-qutrit quantum gates that scales linearly with $n$, the board size.

Overall, we see that all the moves in the classical games of FIR and weiqi obey the principles $\mathbf{P 1}$ and $\mathbf{P 2}$. These classical moves will be a true subset of all the moves in the quantum games.

\section{QUANTUM MOVES}

In each of all the classical moves, the player places one stone at exactly one point. We now construct quantum moves where the player can place one stone simultaneously at different points. As a result, one can view a quantum game as a superposition of finite number classical games, which are played simultaneously. There are infinitely many quantum moves that satisfy the principles $\mathbf{P 1}$ and $\mathbf{P 2}$. For simplicity, we will show only details of how to construct two types of superposition moves and three types of entangled moves. Construction of other quantum moves can be followed straightforwardly.

Hadamard gate is the only one among the universal quantum gates that transforms a non- 
superposition state to a superposition state [1]. A quantum computer with no Hadamard gate is essentially a reversible classical computer. So, to construct quantum moves, we have to use ternary extension of Hadamard gate. There are three of them [2]; we use only two. They are

$$
H^{b}=\frac{1}{\sqrt{2}}\left(\begin{array}{ccc}
1 & 1 & 0 \\
1 & -1 & 0 \\
0 & 0 & \sqrt{2}
\end{array}\right), \quad H^{w}=\frac{1}{\sqrt{2}}\left(\begin{array}{ccc}
\sqrt{2} & 0 & 0 \\
0 & -1 & 1 \\
0 & 1 & 1
\end{array}\right) .
$$

These $H$ gates generate superpositions of a stone and unoccupied at a given point as follows

$$
\begin{aligned}
H^{b}|\boldsymbol{\bigcirc}\rangle & =\frac{1}{\sqrt{2}}(|\boldsymbol{\bigcirc}\rangle+|U\rangle), & H^{b}|U\rangle & =\frac{1}{\sqrt{2}}(|\boldsymbol{\bigcirc}\rangle-|U\rangle) \\
H^{w}|\bigcirc\rangle & =\frac{1}{\sqrt{2}}(|\bigcirc\rangle+|U\rangle), & H^{w}|U\rangle & =\frac{1}{\sqrt{2}}(|\bigcirc\rangle-|U\rangle) .
\end{aligned}
$$

We will use them with other gates to construct quantum moves. These "other gates" include a pair of two-qutrit gates, $B_{(\beta, j)}^{(\alpha, i)}$ and $W_{(\beta, j)}^{(\alpha, i)}$, which are called controlled-X gates in quantum information [2]. If there is a stone at the point $(\alpha, i), B_{(\beta, j)}^{(\alpha, i)}$ takes no action; if there is no stone at the point $(\alpha, i), B_{(\beta, j)}^{(\alpha, i)}$ takes an action of $X^{b}$ at the point $(\beta, j)$. Similarly, if there is a stone at the point $(\alpha, i), W_{(\beta, j)}^{(\alpha, i)}$ takes no action; if there is no stone at the point $(\alpha, i)$, $W_{(\beta, j)}^{(\alpha, i)}$ takes an action of $X^{w}$ at the point $(\beta, j)$. The matrix forms of $B_{(\beta, j)}^{(\alpha, i)}$ and $W_{(\beta, j)}^{(\alpha, i)}$ can be found in Appendix A.

With $H, B$, and $W$ gates, we now introduce superposition moves. A superposition move is to place one stone simultaneously on two unoccupied points. Mathematically, for black stones, they are defined as

$$
S_{(\alpha, i)(\beta, j)}^{b+}=B_{(\beta, j)}^{(\alpha, i)} H_{(\alpha, i)}^{b} X_{(\alpha, i)}^{b}, \quad S_{(\alpha, i)(\beta, j)}^{b-}=B_{(\beta, j)}^{(\alpha, i)} H_{(\alpha, i)}^{b}
$$

One can check that

$$
S_{(\alpha, i)(\beta, j)}^{b \pm}\left|U_{\alpha, i} U_{\beta, j}\right\rangle=\frac{1}{\sqrt{2}}\left(\left|\boldsymbol{\bigcirc}_{\alpha, i} U_{\beta, j}\right\rangle \pm\left|U_{\alpha, i} \boldsymbol{\bigcirc}_{\beta, j}\right\rangle\right)
$$

We can similarly define $S_{(\alpha, i)(\beta, j)}^{w \pm}$ for white stones. One superposition move is illustrated in Fig 4.1 . If we let $|\mathcal{U}\rangle$ represent a board with no stones, the move in Fig.4.1 can be expressed mathematically as

$$
S_{G 3, G 4}^{b+}|\mathcal{U}\rangle=\frac{1}{\sqrt{2}}\left(\left|\boldsymbol{\bigcirc}_{G 3}\right\rangle+\left|\boldsymbol{\bigcirc}_{G 4}\right\rangle\right)
$$

After the superposition move by Bob, we have two games playing at the same time. This is an important feature of superposition move: it doubles the number of games playing. 

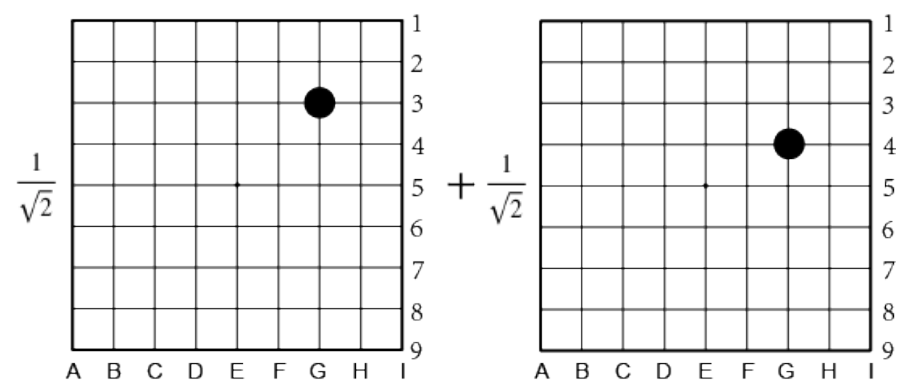

FIG. 4.1: Superposition move. With $S_{G 3, G 4}^{b+}$, Bob places one black stone on the points $(G, 3)$ and $(G, 4)$ simultaneously with equal probability.

When one player makes a move, the other player follows with a counter move. If the previous move is a one-qutrit move, i.e., $X^{b}$ or $X^{w}$, there are two kinds of counter moves. The first kind is called one-to-one counter move $C_{(\beta, j)}^{(\alpha, i)}$, which is essentially a two-qutrit gate: if there is a stone at the point $(\alpha, i)$, then a stone of the opposite color is placed at the point $(\beta, j)$; otherwise, no action is taken. The second kind is called one-to-two counter move $D_{\left(\beta_{1}, j_{1}\right)\left(\beta_{2}, j_{2}\right)}^{(\alpha,)}$ : if there is a stone at the point $(\alpha, i)$, then a stone of the opposite color is placed simultaneously at the points $\left(\beta_{1}, j_{1}\right)$ and $\left(\beta_{2}, j_{2}\right)$ with one of the four superposition moves, $S_{\left(\beta_{1}, j_{1}\right)\left(\beta_{2}, j_{2}\right)}^{b \pm}$ and $S_{\left(\beta_{1}, j_{1}\right)\left(\beta_{2}, j_{2}\right)}^{w \pm}$. It is clear that $D_{\left(\beta_{1}, j_{1}\right)\left(\beta_{2}, j_{2}\right)}^{(\alpha, i) \pm}$ are mathematically three-qutrit gates.

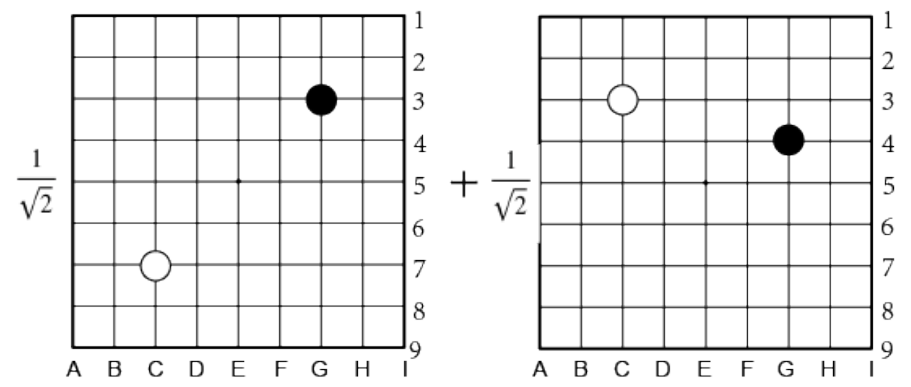

FIG. 4.2: Entangled move: one white stone is placed on two points simultaneously to counter the superposition move of the black stone in Fig 4.1 .

An entangled move is a counter move for a superposition move by the other player. Using two counter moves, we can construct three different types of entangled moves. The first type involves two $C$ moves,

$$
E_{\left(\alpha_{2}, i_{2}\right)-\left(\beta_{2}, j_{2}\right)}^{\left(\alpha_{1}, i_{1}\right)-\left(\beta_{1}, j_{1}\right)}=C_{\left(\beta_{1}, j_{1}\right)}^{\left(\alpha_{1}, i_{1}\right)} C_{\left(\beta_{2}, j_{2}\right)}^{\left(\alpha_{2}, i_{2}\right)}
$$


The second type involves one $C$ move and one $D$ move,

$$
T_{\left(\alpha_{2}, i_{2}\right) \pm\left(\beta_{2}, j_{2}\right)\left(\gamma_{2}, k_{2}\right)}^{\left(\alpha_{1}, i_{1}\right)-\left(\beta_{1}, i_{1}\right)}=C_{\left(\beta_{1}, j_{1}\right)}^{\left(\alpha_{1}, i_{1}\right)} D_{\left(\beta_{2}, j_{2}\right)\left(\gamma_{2}, k_{2}\right)}^{\left(\alpha_{2}, i_{2}\right) \pm}
$$

The third type involves two $D$ moves,

$$
F_{\left(\alpha_{2}, i_{2}\right) \pm\left(\beta_{2}, j_{2}\right)\left(\gamma_{2}, k_{2}\right)}^{\left(\alpha_{1}, i_{1}\right) \pm\left(\beta_{1}, j_{1}\right)\left(\gamma_{1}, k_{1}\right)}=D_{\left(\beta_{1}, j_{1}\right)\left(\gamma_{1}, k_{1}\right)}^{\left(\alpha_{1}, i_{1}\right) \pm} D_{\left(\beta_{2}, j_{2}\right)\left(\gamma_{2}, k_{2}\right)}^{\left(\alpha_{2}, i_{2}\right) \pm}
$$

The first type corresponds to a four-qutrit gate; the second type a five-qutrit gate; the third type a six-qutrit gate. For example, for the superposition move in Fig.4.1, we can counter an entangled move (see Fig 4.2)

$$
E_{C 7, C 3}^{G 3, G 4} \frac{1}{\sqrt{2}}\left(\left|\boldsymbol{\bigcirc}_{G 3}\right\rangle+\left|\boldsymbol{\bigcirc}_{G 4}\right\rangle\right)=\frac{1}{\sqrt{2}}\left(\left|\boldsymbol{\bigcirc}_{G 3} \bigcirc_{C 7}\right\rangle+\left|\boldsymbol{\bigcirc}_{G 4} \bigcirc_{C 3}\right\rangle\right)
$$

In general, there are more quantum moves, for example, using complex coefficients and involving more qutrits. These moves can all be constructed similarly and used in designing board games for quantum computers. We for simplicity have only discussed superposition moves and entangled moves introduced above. It is clear that all these quantum moves obey the principles $\mathbf{P} \mathbf{1}$ and $\mathbf{P} \mathbf{2}$.

We end this section with an example of quantum moves that violate the principle P2. Suppose after a move by Bob the wave function of the board becomes

$$
|\Psi\rangle=\sum_{j=1}^{M} a_{j}\left|\phi_{j}\right\rangle
$$

where $M$ is the total number of games going on and $\left|\phi_{j}\right\rangle$ is one of the games. If the best counter move for $\left|\phi_{j}\right\rangle$ is a white stone at the point $p_{j}$, then the best that Alice can do is to place a white stone simultaneously at the points $p_{j}$ 's, respectively, for all the games $\left|\phi_{j}\right\rangle$ 's. This is similar to entangled moves but at much larger scales. To do it, Alice have to select a group of points on the board, whose states are distinct for every $\left|\phi_{j}\right\rangle$, and use them as the control qutrits to make the move. If this group has $G$ points, the total number of qutrits involved is around $G+N_{M}$, where $N_{M}$ is the number of different $p_{j}$ 's. In the late stage of the match, both $G$ and $N_{M}$ should scale with the total number of points on the board. As many of the qutrits are used as control qutrits, the involved qutrits are clearly correlated and thus violate the principle P2. Since this kind of quantum move is made by considering distinct features of every game $\left|\phi_{j}\right\rangle$, we call it game-wise move. All the game-wise moves 
clearly violate the principle $\mathbf{P 2}$. Since each $p_{j}$ is, respectively, the best move for $\left|\phi_{j}\right\rangle$, the principle P2 implies that a quantum computer is inherently incapable of executing the best move possible when the number of superposed games is exponentially large.

\section{GAME OF FIR FOR QUANTUM COMPUTER}

The basic rules of the classical game of FIR (Five in a Row) are as follows. Alice and Bob take turn to place stones on a $15 \times 15$ board with Bob, who has black stones, playing first. The stones can only be placed on unoccupied points. The player who has managed first to have five stones of the same color in a row (horizontal, vertical, or diagonal) wins. Only one-qutrit gates are used in this classical game.

The basic rules of FIR for quantum computers are as follows. The board size is chosen to be the largest allowed by the quantum computer. Alice and Bob take turn to make moves with Bob playing first. There are now quantum moves available, for example, superposition moves and entangled moves discussed above. As a result, there are more than one games going on in the perspective of classical players. For example, if Bob makes a first move as in Fig. 4.1 and Alice follows with an entangled move in Fig. 4.2. We have two games playing simultaneously. In general, the state of the board should have a form as in Eq.(4.11) with a total of $M$ games playing simultaneously. We define the rule of winning as follows. If there are five black stones in a row in any of the games $\left|\phi_{j}\right\rangle$, Bob wins; if five white stones in a row in any $\left|\phi_{j}\right\rangle$, Alice wins.

There are three different schemes to play quantum FIR. The first scheme, denoted as QwQ, is between two quantum computers Alice and Bob, who take turn to make moves. The board state is stored on both quantum computers. After one player makes a move, the updated board state is transferred by a quantum communication network to the other player. This QwQ scheme is illustrated in Fig.5.1. There are at least two enormous challenges to realize this scheme for quantum games. The first challenge is of theoretical nature: it is not clear how a quantum computer itself can assess the quantum state of the board and decide what move to make next. The second is a technical challenge. So far, there are no universal fault-tolerant quantum computers and people have only realized the communication of the quantum state of a single qubit whereas the state of the board is a multiqutrit quantum state. 


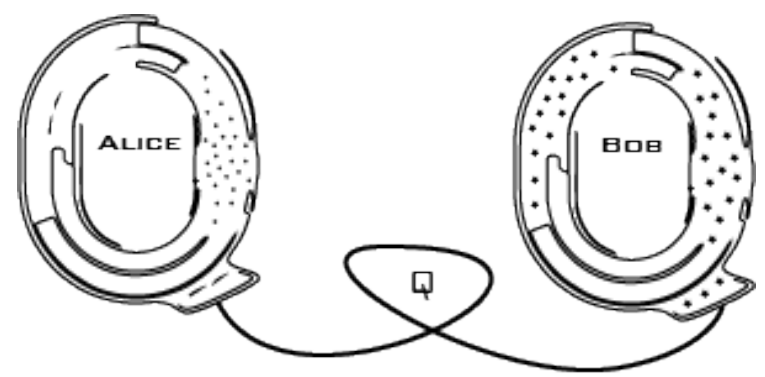

FIG. 5.1: Quantum computer Alice plays a board game with another quantum computer Bob. The state of the board game, which is a multiqutrit quantum state, is transferred by a quantum communication network between them.

The second scheme, called CQC, is that two classical computers Alice and Bob play a quantum game on a quantum computer (see Fig.5.2). The state of the board is stored on the quantum computer. Whenever, a classical computer, say, Alice, makes a move, she executes the corresponding quantum gates on the quantum computer and updates the state of board. At the same time, she communicates her move, which is classical information, to Bob. In this scheme, there is also a theoretical challenge, how a classical computer assesses the quantum state of the board. The classical computer can accomplish this by measurement. However, this is destructive and, more importantly, it becomes impractical when the number of superposed games $M$ is exponentially large. In particular, one is interested to know whether there are five stones of the same color in a row in the board state and whether it is possible to do it without using destructive measurement. Note that this challenge is different from the one in QwQ, where the quantum computer tries to assess its own state.

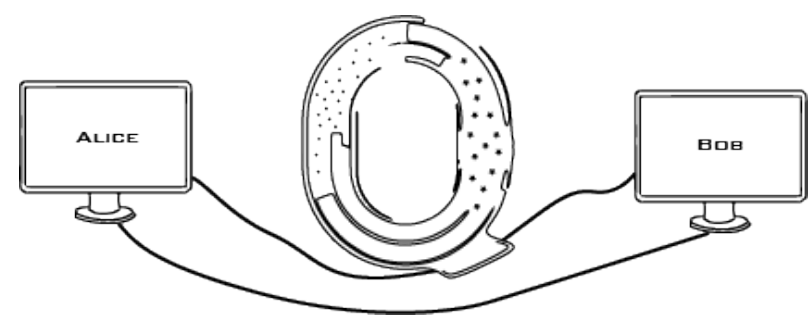

FIG. 5.2: Classical computer Alice plays a quantum board game with another classical computer Bob on a quantum computer. The two classical computers execute quantum gates in turn on the quantum computer and update the state of the board. At the same time they communicate classically between them about the moves. 
In principle, we can simulate any quantum system on a classical computer if the classical computer has exponentially large amount of physical memory. Therefore, two classical computers, Alice and Bob, can also play a quantum board game. This scheme is called $\mathrm{CwC}$ and is shown in Fig 5.3 . However, since we do not have exponentially large memory in reality, we will have to limit the number of superposed games when the quantum game is played by two classical computers. Suppose that the upper limit is $J$, which is independent of the board size. Then after some quantum moves by both players, the board state will reach the limit and become

$$
|\Psi\rangle=\sum_{j=1}^{J} b_{j}\left|\phi_{j}\right\rangle .
$$

All these games $\left|\phi_{j}\right\rangle$ are stored on the classical computers along with $b_{j}$ 's. After this limit, both Alice and Bob are only allowed to make classical moves or game-wise moves. Here the game-wise moves are allowed because the number of games $J$ is fixed and does not scale with the board size. With this limitation, the scheme $\mathrm{CwC}$ is clearly feasible.

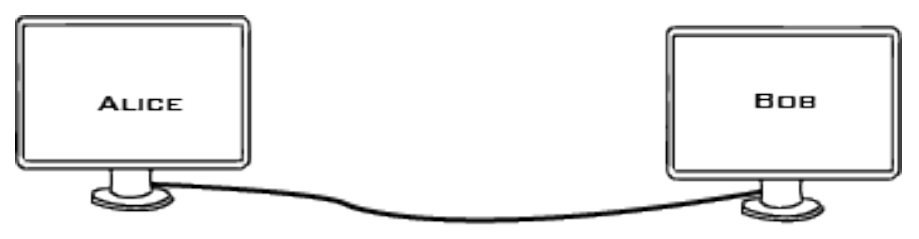

FIG. 5.3: Classical computer Alice plays a quantum board game with another classical computer Bob. In this case, the quantum state of board is stored classically on both two classical computers. Alice and Bob communicate classically between them about the moves.

Let us now re-consider the scheme CQC. It is possible that $\mathrm{CQC}$ is equivalent to $\mathrm{CwC}$. Here is the explanation. In CQC, the board state $|\Psi\rangle$ on the quantum computer is uniquely determined by the sequence of quantum moves made alternatively by Alice and Bob since $|\Psi\rangle$ can be written as

$$
|\Psi\rangle=Q_{Z}^{w} Q_{Z-1}^{b} \cdots Q_{j+1}^{w} Q_{j}^{b} \cdots Q_{2}^{w} Q_{1}^{b}|\mathcal{U}\rangle,
$$

where $Q_{j+1}^{w}$ and $Q_{j}^{b}$ are quantum moves made, respectively, by Alice and Bob. This sequence of $Q^{w}$ 's and $Q^{b}$ 's are classical information but they uniquely determine the games $\left|\phi_{j}\right\rangle$ and their coefficients $a_{j}$ in Eq.4.11. Note that the number of $Q^{w}$ 's and $Q^{b}$ 's is only polynomially large while $M$, the number of games $\left|\phi_{j}\right\rangle$, can be exponentially large. This shows an 
interesting fact that a quantum state, which has too many components to be stored directly on a classical compute, can still be coded as classical information of polynomial size. Both of Alice and Bob are aware of the sequence of $Q^{w}$ and $Q^{b}$. If they can extract information about the games $\left|\phi_{j}\right\rangle$ from $Q^{w}$ and $Q^{b}$ and decide what is the best next move, then we would no longer need the quantum computer to store $\left|\phi_{j}\right\rangle$ and their coefficients $a_{j}$. The two

classical computers, Alice and Bob, in principle can play the quantum game by knowing just the sequence of $Q^{w}$ and $Q^{b}$. As a result, CQC would become CwC. The problem at this moment is that it is not clear how one can extract information about the games $\left|\psi_{j}\right\rangle$ from $Q^{w}$ and $Q^{b}$. One possibility is to use machine learning.

The above discussion may appear general, not limited to the game of FIR. In a way, it is. However, as we will see in the next section, if the quantum game of weiqi is played in both QwQ and CQC, it may never finish. Such a possibility for FIR is very remote.

\section{GAME OF WEIQI FOR QUANTUM COMPUTER}

The key difference of weiqi from FIR is that there is capture of stones of the opposite color. To discuss the quantum game of weiqi, we need to generalize capture quantum mechanically. However, this generalization is highly non-trivial. There are at least two possible approaches. In the first approach, when a stone is captured in one game, it is removed from all the games. One example is shown Fig. 6.1, where the black stone with 3 is captured in the right game and it is removed from both the left and right games. In the second approach as illustrated in Fig. 6.2, the stone is removed only from the games where it is captured and stays in the games where it is still alive. For the first approach, if the stone was placed on the board by a classical move or a superposition move, it can be removed by executing the inverse move. However, if the stone was placed with one of the entangled moves, its removal becomes difficult. As the stone to be removed has different surrounding in different games $\left|\phi_{j}\right\rangle$, thus the removal is a game-wise move. In the second approach, it is obvious that the removal of the stone is a game-wise move as one has to examine every game to determine whether the stone should be removed. Therefore, both approaches violate the principle P2.

To satisfy the principle $\mathbf{P 2}$, we have to adopt an approach of capture that appears odd but is fundamentally logical. We use the example in Fig 6.3 to illustrate. Before the eighth 


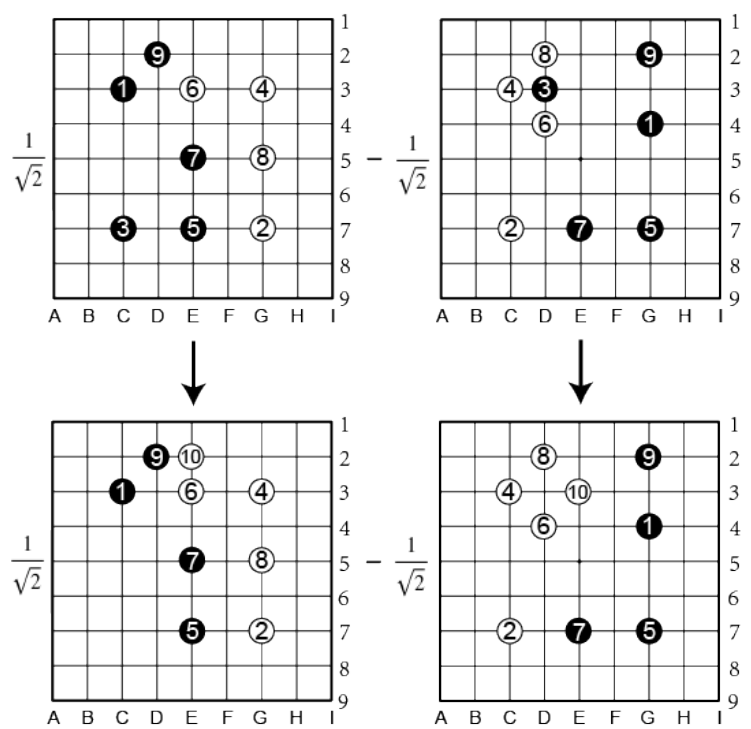

FIG. 6.1: One possible way of capture a stone in the quantum game of weiqi: the captured stone is removed from all the games playing in parallel. The numbers on the stones mark the order that the stones are placed on the board: the black stone with 1 is the first stone placed on the board, the white stone with 2 is the second placed, etc.

stone, which is white, is placed on the board, the wave function of the board is

$$
\left|\Phi_{0}\right\rangle=\frac{1}{\sqrt{2}}\left(\left|\bigcirc_{C 3} \bigcirc_{G 7} \bigcirc_{C 7} \bigcirc_{G 3} \bigcirc_{F 7} \bigcirc_{F 6} \bigcirc_{E 7}\right\rangle-\left|\bigcirc_{G 4} \bigcirc_{G 5} \bigcirc_{D 3} \bigcirc_{H 4} \bigcirc_{C 7} \bigcirc_{G 3} \bigcirc_{G 7}\right\rangle\right)
$$

After the eighth stone is placed by Alice with an entangled move, the first stone, which is black, is captured in the game on the right side. This triggers a mandatory move, removing the first stone from the right game. Mathematically, this is given by $X_{G 4}^{b}$. This means that, if we apply this mandatory move to every game, we have $\left|\Phi_{0}\right\rangle$ as follows

$$
\begin{aligned}
\left|\Phi_{1}\right\rangle=X_{G 4}^{b} E_{G 7-F 4}^{E 7-E 5}\left|\Phi_{0}\right\rangle= & \frac{1}{\sqrt{2}}\left(\left|\bigcirc_{C 3} \bigcirc_{G 7} \bigcirc_{C 7} \bigcirc_{G 3} \bigcirc_{F 7} \bigcirc_{F 6} \bigodot_{E 7} \bigcirc_{E 5} \bigcirc_{G 4}\right\rangle\right. \\
& \left.-\left|\bigcirc_{G 5} \bigcirc_{D 3} \bigcirc_{H 4} \bigcirc_{C 7} \bigcirc_{G 3} \bigcirc_{G 7} \bigcirc_{F 4}\right\rangle\right)
\end{aligned}
$$

The result is, of course, odd and surprising to people who are familiar to the game of weiqi: for the game on the left side, there is a black stone with even number and it is in a way placed there by Alice. However, it is fundamentally logical and does not violate the principle P2. There may be other ways to generalize capture to the quantum game of weiqi. This approach appears to the simplest and most straightforward. It is important to note the effects of $X_{G 4}^{b}$ : it removes it if there is a black stone at the point $(G, 4)$; it places a black 


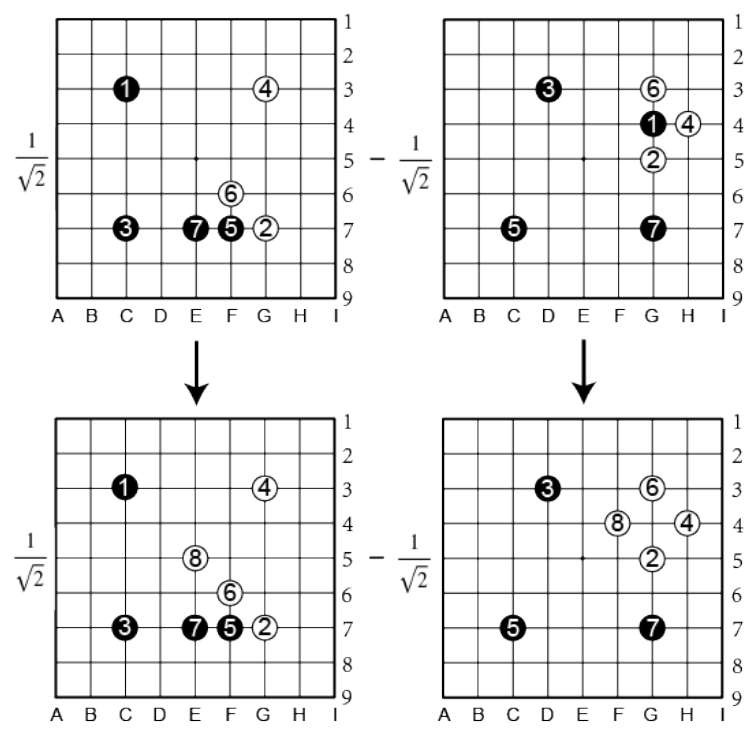

FIG. 6.2: Another possible way of capture a stone in the quantum game of weiqi: the stone is removed from the game where it is captured and stays in the other game. The numbers on the stones mark the order that the stones are placed on the board: the black stone with 1 is the first stone placed on the board, the white stone with 2 is the second placed, etc.

stone at the point $(G, 4)$ if it is unoccupied; it has no effect if there is a white stone at the point $(G, 4)$. Its first two effects are illustrated in Fig 6.3 . The third effect means that if there is a white stone at the point $(G, 4)$ in the left game, it stays there.

In the traditional game of weiqi, there could be unending cycles of captures by both players. One example is shown in Fig.6.4 after the marked white stone is captured by the black, Alice can place a white stone at position B to capture the marked black stone; then the marked white stone can be captured again by Bob. To prevent this kind of fruitless unending cycle, there is the ko rule. For the situation in Fig,6.4, after the marked white stone is captured, the ko rule forbids Alice to capture the marked black stone immediately, and Alice has to make at least one different move in other parts of the board before being allowed to capture the marked black stone. It is very unlikely that there are unending cycles in the quantum game of weiqi for two reasons: (1) there are usually multiple games going in parallel; (2) the quantum capture is very different from its classical counterpart when there are multiple games simultaneously.

In the game of weiqi, there are forbidden points where a stone of a certain color is not allowed. For example, in the lower right game of Fig. 6.3 , the point $(G, 4)$ is forbidden for 


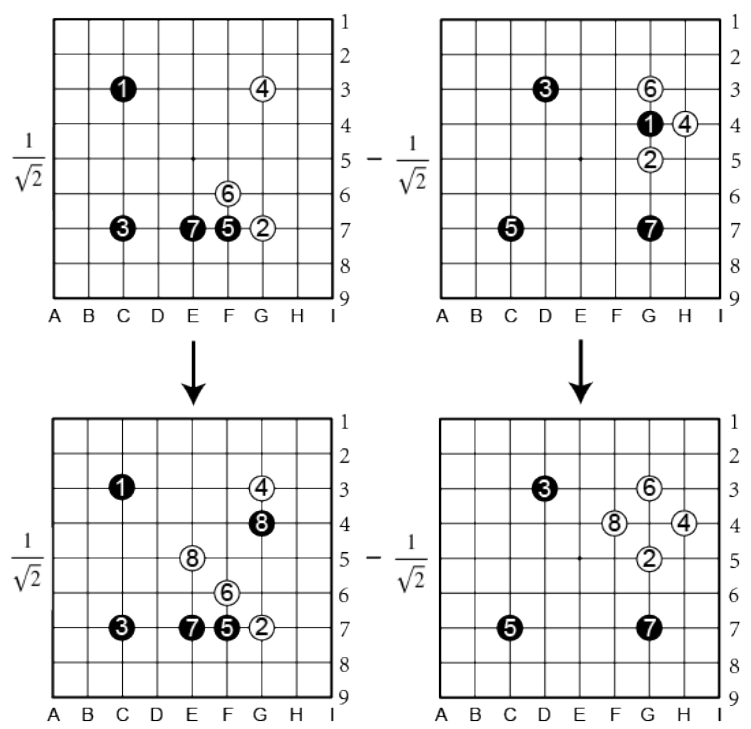

FIG. 6.3: A legitimate way of capture a stone in the quantum game of weiqi: the stone at a certain position is removed from the game when it is captured and a stone of the same color appears at the same position in the other game. The numbers on the stones mark the order that the stones are placed on the board: the black stone with 1 is the first stone placed on the board, the white stone with 2 is the second placed, etc.

black stone.

We are now ready to state the basic rules of weiqi for quantum computers: Alice and Bob take turn to make either classical or quantum moves with Bob playing first. In each move, a stone can not be placed at forbidden points. When an unending cycle appears, the ko rule is enforced.

Same as FIR, there are three schemes to play the quantum game of weiqi. However, both the schemes $\mathrm{QwQ}$ and $\mathrm{CQC}$ are not very meaningful for weiqi. The reason is that the game of weiqi is much more complicated. In an ordinary classical game of weiqi, there are usually more than 200 moves near the end. This means that there are more than 200 stones on a $19 \times 19$ board. Therefore, for the quantum game of weiqi, at the late stage of a match. we expect similarly that there are around 200 stones on the board and, in addition, there are exponentially large number of games going on simultaneously. Suppose that Bob now wants to make a classical move, placing a stone at a given point. However, this given point at this stage is very unlikely unoccupied for all the games $\left|\psi_{j}\right\rangle$. As a result, this move by Bob can remove a black stone in some games $\left|\psi_{j}\right\rangle$. It is well known in the game of weiqi, if the 

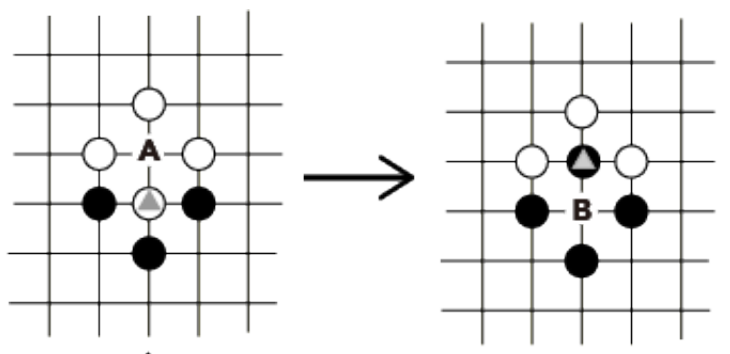

个
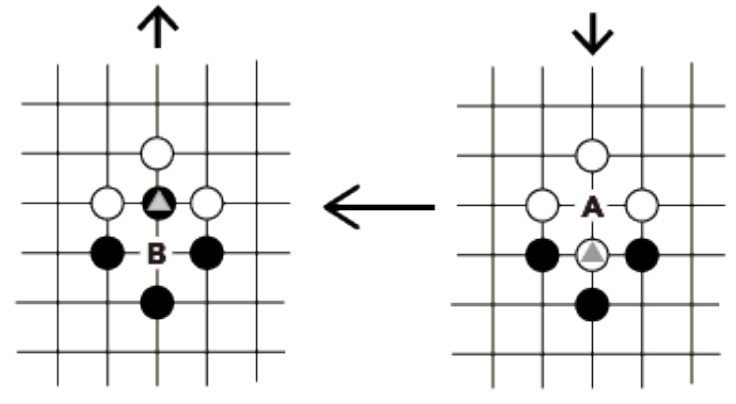

FIG. 6.4: Ko rule for unending cycle of captures. According to this rule, after the capture of the marked white stone (upper left), Alice is not allowed to place a white stone on position B to capture the marked black stone. She has to wait for at least one move.

number of stones keep reducing, the game may never finish. To make legitimate moves in the late stage of the weiqi game, one has to use game-wise moves, which violate the principle P2. There is also such a possibility for FIR. However, FIR usually does not last that long and will likely to end before there are too many stones on the board. It would be very interesting if one could design a new and competitive board game for quantum computer which has no shortcoming discussed above.

For the game of weiqi, the scheme $\mathrm{CwC}$ is also feasible when we impose limitation on the number of superposed games. We have already noted in the discussion of FIR that game-wise moves are allowed in this scheme. It is similar here for the game of weiqi with an interesting twist: we use either the quantum capture illustrated in Fig 6.1 or the one illustrated in Eq. (6.2) and Fig 6.3. We do not use the capture shown in Fig 6.2. This is to keep the games in Eq. (5.1) related. In the game of FIR, when the limit is reached, it becomes clear that the best strategy is to examine every game $\left|\phi_{j}\right\rangle$ in Eq. (5.1), find its best move $p_{j}$, and make a game-wise move, that is, placing a stone simultaneously at points $p_{j}$ 's, respectively, for games $\left|\phi_{j}\right\rangle$ 's. As a result, the games $\left|\phi_{j}\right\rangle$ become independent of each other. In the game of weiqi, if we adopt the capture shown in Fig 6.2, the games $\left|\phi_{j}\right\rangle$ would also become independent of each other. It is clearly more interesting to keep the games related. 
For this reason, we use either of the other two approaches of capture in the scheme $\mathrm{CwC}$. In fact, we prefer the approach of Fig.6.1 since the one in Fig.6.3 may appear too odd for traditional players.

\section{DISCUSSION AND CONCLUSION}

We have discussed the three principles for designing board games for quantum computer. The principle $\mathbf{P 2}$ is particularly interesting and important as it exposes an inherent inability of a quantum computer to execute the best possible move. With two examples, Five in a Row (FIR) and weiqi, we have shown the details to construct quantum moves that obey these three principles. There have been some work discussing board games with quantum features [4, 5]. The closest to our work is the discussion of quantum chess in Ref. [4, 5], where it is stressed that moves should be unitary. However, nothing similar to our principle P2 is discussed as chess is not a scalable board game. The generalized game of weiqi (or go) in Ref.[4] has some quantum features. But its moves are not always unitary and the generalized game can not be played on or by quantum computer.

We have also discussed three different schemes to play the quantum games. The third scheme $\mathrm{CwC}$ is technically feasible and can be readily programmed. The other two schemes not only face daunting technical challenges as they involve yet-to-be-built universal quantum computer, but also need to overcome some theoretical issues. The future study of board games for quantum computer, in particular with the scheme QwQ, may lead to the understanding of how intelligence emerges out of the quantum world.

\section{Acknowledgments}

We dedicate this work to Professor P. W. Anderson, who was a very good amateur weiqi (or go) player and would probably view a quantum game of weiqi as a spin-1 system on a square lattice. This work is supported by the The National Key R\&D Program of China (Grants No. 2017YFA0303302, No. 2018YFA0305602), National Natural Science Foundation of China (Grant No. 11921005), and Shanghai Municipal Science and Technology Major Project (Grant No.2019SHZDZX01). 


\section{Appendix A: Matrix forms of controlled quantum gates $B_{(\beta, j)}^{(\alpha, i)}$ and $W_{(\beta, j)}^{(\alpha, i)}$}

Here we give the explicit matrix forms for quantum gates $B_{(\beta, j)}^{(\alpha, i)}$ and $W_{(\beta, j)}^{(\alpha, i)}$ introduced in Section IV. If there is a stone at the point $(\alpha, i), B_{(\beta, j)}^{(\alpha, i)}$ takes no action; if there is no stone at the point $(\alpha, i), B_{(\beta, j)}^{(\alpha, i)}$ takes an action of $X^{b}$ at the point $(\beta, j)$. Following the conventional tensor rule, we can write $B_{(\beta, j)}^{(\alpha, i)}$ as a $9 \times 9$ matrix

$$
B_{(\beta, j)}^{(\alpha, i)}=\left(\begin{array}{ccccccccc}
1 & 0 & 0 & 0 & 0 & 0 & 0 & 0 & 0 \\
0 & 1 & 0 & 0 & 0 & 0 & 0 & 0 & 0 \\
0 & 0 & 1 & 0 & 0 & 0 & 0 & 0 & 0 \\
0 & 0 & 0 & 0 & 1 & 0 & 0 & 0 & 0 \\
0 & 0 & 0 & 1 & 0 & 0 & 0 & 0 & 0 \\
0 & 0 & 0 & 0 & 0 & 1 & 0 & 0 & 0 \\
0 & 0 & 0 & 0 & 0 & 0 & 1 & 0 & 0 \\
0 & 0 & 0 & 0 & 0 & 0 & 0 & 1 & 0 \\
0 & 0 & 0 & 0 & 0 & 0 & 0 & 0 & 1
\end{array}\right)
$$

The move $W_{(\beta, j)}^{(\alpha, i)}$ is the counterpart for white stones and it has the following matrix form,

$$
W_{(\beta, j)}^{(\alpha, i)}=\left(\begin{array}{ccccccccc}
1 & 0 & 0 & 0 & 0 & 0 & 0 & 0 & 0 \\
0 & 1 & 0 & 0 & 0 & 0 & 0 & 0 & 0 \\
0 & 0 & 1 & 0 & 0 & 0 & 0 & 0 & 0 \\
0 & 0 & 0 & 1 & 0 & 0 & 0 & 0 & 0 \\
0 & 0 & 0 & 0 & 0 & 1 & 0 & 0 & 0 \\
0 & 0 & 0 & 0 & 1 & 0 & 0 & 0 & 0 \\
0 & 0 & 0 & 0 & 0 & 0 & 1 & 0 & 0 \\
0 & 0 & 0 & 0 & 0 & 0 & 0 & 1 & 0 \\
0 & 0 & 0 & 0 & 0 & 0 & 0 & 0 & 1
\end{array}\right) .
$$

\section{Appendix B: Absence of interference in quantum board games}

Interference is a hallmark of quantum phenomena. In general, it can also exist in quantum board games. However, in our quantum board games, if we limit ourselves to the classical moves, superposition and entangled moves introduced in Section IV, interference does not exist. Here is the proof. 
Without loss of generality, we consider the state of the board after one of Alice's moves

$$
|\Psi\rangle=a_{1}\left|\phi_{1}\right\rangle+a_{2}\left|\phi_{2}\right\rangle+\sum_{j=3}^{M} a_{j}\left|\phi_{j}\right\rangle .
$$

Assume that interference happens here for the first time, say, $\left|\phi_{1}\right\rangle=\left|\phi_{2}\right\rangle$. We will show that if this is the case, it would lead to contradiction. Let

$$
|\tilde{\Psi}\rangle=\sum_{j=3}^{\tilde{M}} \tilde{a}_{j}\left|\tilde{\phi}_{j}\right\rangle
$$

be the state of board before the Alice's move. There must be at least two games $\left|\tilde{\phi}_{j_{1}}\right\rangle$ and $\left|\tilde{\phi}_{j_{2}}\right\rangle$, which become $\left|\phi_{1}\right\rangle$ and $\left|\phi_{2}\right\rangle$, respectively, after the Alice's move. That Alice's move only adds a white stone on the board has two implications: (1) the black stones in both $\left|\tilde{\phi}_{j_{1}}\right\rangle$ and $\left|\tilde{\phi}_{j_{2}}\right\rangle$ must be exactly the same; (2) the white stones are different. The former means that Alice's move can not be an entangled move; the latter implies that her move can not a classical move, either.

Alice's last choice is a superposition move that places a white stone to both positions $p_{1}$ and $p_{2}$. Assume that $\left|\tilde{\phi}_{j_{1}}\right\rangle$ turns to $\left|\phi_{1}\right\rangle$ with a white stone at $p_{1}$ and $\left|\tilde{\phi}_{j_{2}}\right\rangle$ turns to $\left|\phi_{2}\right\rangle$ with a white stone at $p_{2}$. With $\left|\phi_{1}\right\rangle=\left|\phi_{2}\right\rangle$, there must be a white stone at $p_{2}$ in the game $\left|\tilde{\phi}_{j_{1}}\right\rangle$ and a white stone at $p_{1}$ in the game $\left|\tilde{\phi}_{j_{2}}\right\rangle$. This means that the superposition move that places a white stone to both positions $p_{1}$ and $p_{2}$ is not legitimate. Therefore, Alice's last choice is also impossible.

Based on the above discussion, we find that there is interference in our quantum board games if we limit ourselves to the classical moves, superposition moves and entangled moves.

[1] M. A. Nielsen and I. L. Chuang, Quantum Computation and Quantum Information (Cambridge University Press, Cambridge, 2000).

[2] Y.-M. Di and H.-R. Wei, arXiv:1105.5485 (2012).

[3] D. Aharonov, W. van Dam, J. Kempe, Z. Landau, S. Lloyd, and O. Regev, SIAM J. Comput. 37, 166 (2007).

[4] A. Ranchin, arXiv:1603.04751 (2016).

[5] C. Cantwell, arXiv:1906.05836 (2019). 